Delft University of Technology

\title{
Reconstitution of Ultrawide DNA Origami Pores in Liposomes for Transmembrane Transport of Macromolecules
}

Fragasso, Alessio; De Franceschi, Nicola; Stömmer, Pierre; Van Der Sluis, Eli O.; Dietz, Hendrik; Dekker, Cees

DOI

10.1021/acsnano.1c01669

Publication date

2021

Document Version

Final published version

Published in

ACS Nano

\section{Citation (APA)}

Fragasso, A., De Franceschi, N., Stömmer, P., Van Der Sluis, E. O., Dietz, H., \& Dekker, C. (2021).

Reconstitution of Ultrawide DNA Origami Pores in Liposomes for Transmembrane Transport of

Macromolecules. ACS Nano, 15(8), 12768-12779. https://doi.org/10.1021/acsnano.1c01669

\section{Important note}

To cite this publication, please use the final published version (if applicable).

Please check the document version above.

\footnotetext{
Copyright

Other than for strictly personal use, it is not permitted to download, forward or distribute the text or part of it, without the consent of the author(s) and/or copyright holder(s), unless the work is under an open content license such as Creative Commons.

Takedown policy

Please contact us and provide details if you believe this document breaches copyrights.

We will remove access to the work immediately and investigate your claim.
} 


\title{
Reconstitution of Ultrawide DNA Origami Pores in Liposomes for Transmembrane Transport of Macromolecules
}

\author{
Alessio Fragasso, Nicola De Franceschi, Pierre Stömmer, Eli O. van der Sluis, Hendrik Dietz,* \\ and Cees Dekker*
}

Cite This: https://doi.org/10.1021/acsnano.1c01669

Read Online

\section{ACCESS}

山ll Metrics \& More

Article Recommendations

Supporting Information

ABSTRACT: Molecular traffic across lipid membranes is a vital process in cell biology that involves specialized biological pores with a great variety of pore diameters, from fractions of a nanometer to $>30 \mathrm{~nm}$. Creating artificial membrane pores covering similar size and complexity will aid the understanding of transmembrane molecular transport in cells, while artificial pores are also a necessary ingredient for synthetic cells. Here, we report the construction of DNA origami nanopores that have an inner diameter as large as $30 \mathrm{~nm}$. We developed methods to successfully insert these ultrawide pores into the

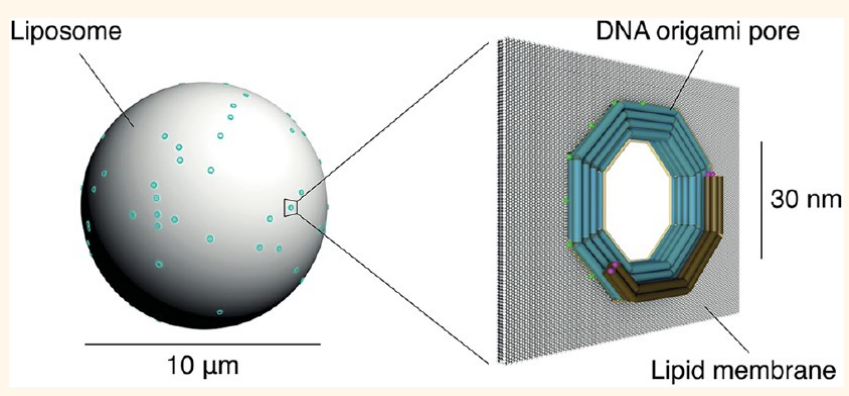
lipid membrane of giant unilamellar vesicles (GUVs) by administering the pores concomitantly with vesicle formation in an inverted-emulsion cDICE technique. The reconstituted pores permit the transmembrane diffusion of large macromolecules, such as folded proteins, which demonstrates the formation of large membrane-spanning open pores. The pores are size selective, as dextran molecules with a diameter up to 28 $\mathrm{nm}$ can traverse the pores, whereas larger dextran molecules are blocked. By FRAP measurements and modeling of the GFP influx rate, we find that up to hundreds of pores can be functionally reconstituted into a single GUV. Our technique bears great potential for applications across different fields from biomimetics, to synthetic biology, to drug delivery.

KEYWORDS: DNA origami, nanopores, liposomes, transmembrane transport, cDICE

\section{INTRODUCTION}

In the past decade, advancements in the field of DNA nanotechnology have enabled the fabrication of a great variety of "DNA origami" nanostructures, ${ }^{1,2}$ including transmembrane structures that resemble the biological pores found in cells. ${ }^{3}$ Drawing inspiration from protein pores such as $\alpha$-hemolysin, ${ }^{4}$ artificial pores have been engineered with DNA origami ${ }^{1,2}$ that can insert into lipid bilayers and allow for transmembrane diffusion of ions ${ }^{5,6}$ and small molecules such as fluorophores, ${ }^{7,8}$ DNA oligomers, ${ }^{5,7}$ short PEG, ${ }^{9}$ and dextran. ${ }^{8,10}$ To favor partitioning into the lipid bilayer, a popular strategy relies on the chemical modification of the outer nanopore surface with hydrophobic groups, such as cholesterols, that anchor and stabilize the strongly hydrophilic DNA-based nanopores into the lipid membrane. ${ }^{3}$ As DNA origami structures can be designed with virtually any shape or $\operatorname{size}^{11}$ up to the dimensions comparable to those of viruses, ${ }^{12}$ the approach bears great potential for various applications, from recapitulating the function of complex enzymes like flippase, ${ }^{13}$ to mimicking large protein transporters such as the nuclear pore complex. $^{14,15}$

However, so far, only small pores with an inner diameter of a few nanometers have been realized, ${ }^{8,10}$ which is because wider pores are increasingly difficult to insert into a membrane. A commonly used strategy to reconstitute pores into a bilayer relies on spontaneous insertion into a preformed lipid membrane. ${ }^{3}$ This method relies on transient membrane instabilities $^{16}$ and becomes quickly ineffective for larger size objects. This is because the work required to open up a hole in a membrane grows quadratically with the diameter of the hole, ${ }^{6,8,17}$ and spontaneous creation of such a hole occurs in a Boltzmann weighted fashion, which exponentially decreases

Received: February 24, 2021

Accepted: June 22, 2021 

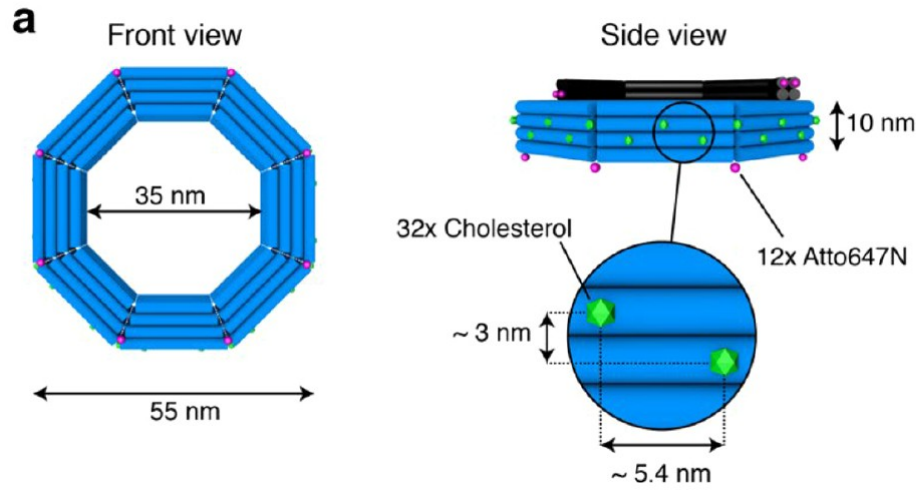

b

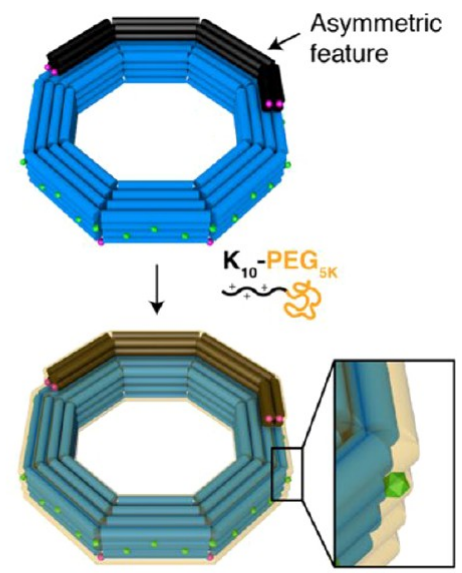

C

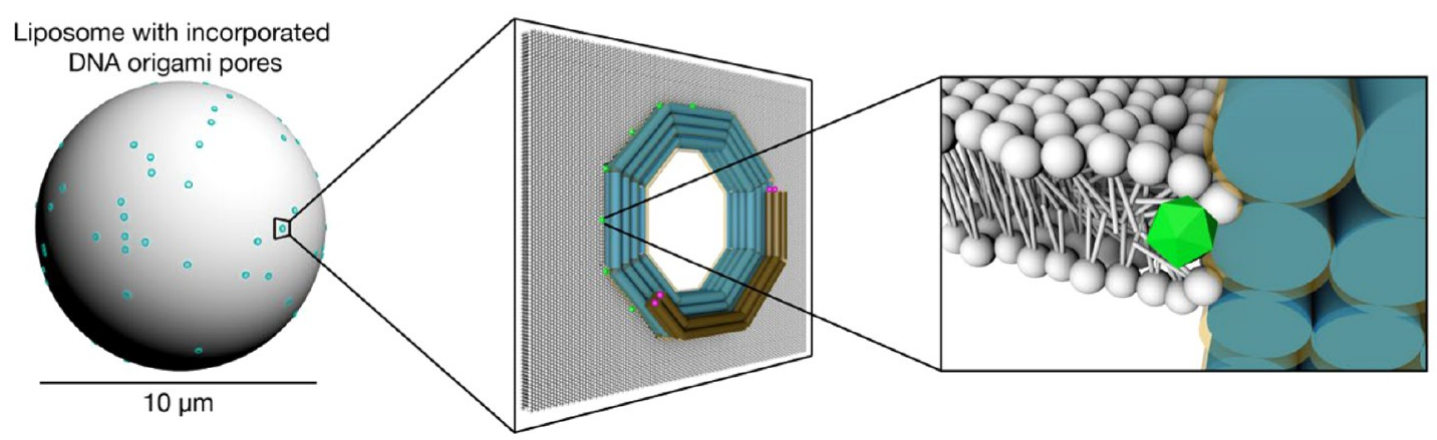

Figure 1. DNA origami pore design and incorporation approach. (a) DNA origami pore in front (left) and side view (right). Blue cylinders represent DNA double helices. The outer diameter is $55 \mathrm{~nm}$, and the inner diameter is $35 \mathrm{~nm}$. Green objects along the outer side of the DNA origami pore indicate the 32 cholesterol modifications. They vertically span over a width of $\sim 3$ nm (purposely slightly less than the hydrophobic thickness of $\sim 3.7 \mathrm{~nm}$ of a DOPC lipid bilayer ${ }^{37}$ ). Black cylinders represent an asymmetric feature that was introduced for class averaging of single particles from TEM. Violet spots along the outer surface of the DNA origami pore indicate the positions of $12 \mathrm{Atto647N}$ dye modifications. The pore is designed with a height of $10 \mathrm{~nm}$. (b) Schematic representation of $\mathrm{K}_{10}-\mathrm{PEG}_{5 \mathrm{~K}}$ coating of the origami pore. (c) Schematic representation of the DNA origami pore incorporated into the lipid bilayer of a GUV. Left: GUV with pores incorporated in the lipid bilayer. Middle: zoom-in on one of the incorporated origami pores resulting in a $\mathbf{3 0} \mathrm{nm}$ diameter hole. Right: further zoom-in showing individual lipid molecules of the bilayer; white spheres represent lipid heads, white strokes represent lipid tails, and the green object represents a cholesterol.

the chances of success for inserting large pores into spontaneously occurring membrane defects. Scaling up the size of artificial pores thus requires an alternative insertion process that can circumvent the barriers associated with spontaneous insertion.

Here, we overcome these challenges by employing a continuous droplet interface crossing encapsulation (cDICE) technique $^{18}$ to incorporate ultrawide $(\sim 30 \mathrm{~nm}$ inner diameter, $\sim 55 \mathrm{~nm}$ outer diameter) DNA origami pores into the membrane of giant unilamellar vesicles (GUVs). As we show below, by administering the pores concomitantly with vesicle formation, the pores can be localized efficiently at the membrane. To demonstrate the transmembrane transport through the pores, we measured the influx of multiple fluorescent macromolecules with different sizes using confocal microscopy. We find that the origami pores indeed support the transit of large (up to $28 \mathrm{~nm}$ ) molecular structures, consistent with the $30 \mathrm{~nm}$ inner diameter of the pores. Using fluorescence recovery after photobleaching (FRAP), we estimate that up to hundreds of pores can be functionally reconstituted in a single liposome. These ultrawide pores represent an exciting tool for various applications, from the mimicking of large biological pores such as the nuclear pore complex to applications in synthetic biology and drug delivery.

\section{RESULTS AND DISCUSSION}

Design and Assembly of Ultrawide DNA Origami Pores. Using DNA origami folding of a template strand, ${ }^{1,2}$ we designed and assembled a rigid octagonal ring-like structure (see Figure 1). A multilayer DNA origami object was designed in square-lattice helical packing and folded from a 7560 bases long scaffold single strand with 240 individual oligonucleotide single strands, arranged in a $4 \times 4$ double-helical pattern. It was designed to form a closed-loop octagonal shape, formed by 8 corner design motifs ${ }^{19}$ with single-stranded poly-T strands at the corner sites. Deletions were introduced every 32 bases to correct for global twist. ${ }^{20}$ The origami pore was designed with a nominal outer diameter of $55 \mathrm{~nm}$, an inner diameter of 35 $\mathrm{nm}$, and a height of $10 \mathrm{~nm}$ (Figure 1a), which represents the channel length. The design includes 48 single-stranded handles that were distributed evenly on the interior surface facing the central cavity, as sites for future functionalization purposes (Figure S15). The octagon furthermore included 32 cholesterol modifications on the outer surface that were arranged evenly with a $\sim 5.5 \mathrm{~nm}$ horizontal spacing and $\sim 3 \mathrm{~nm}$ vertical distance between neighboring cholesterols (Figure 1a). An asymmetric feature was added (Figure 1b) consisting of four additional double helices on top of the octagon, arranged in a $2 \times 2$ double helical pattern on the top side of the octagon, spanning over 3 corners. The octagon further features 12 
a

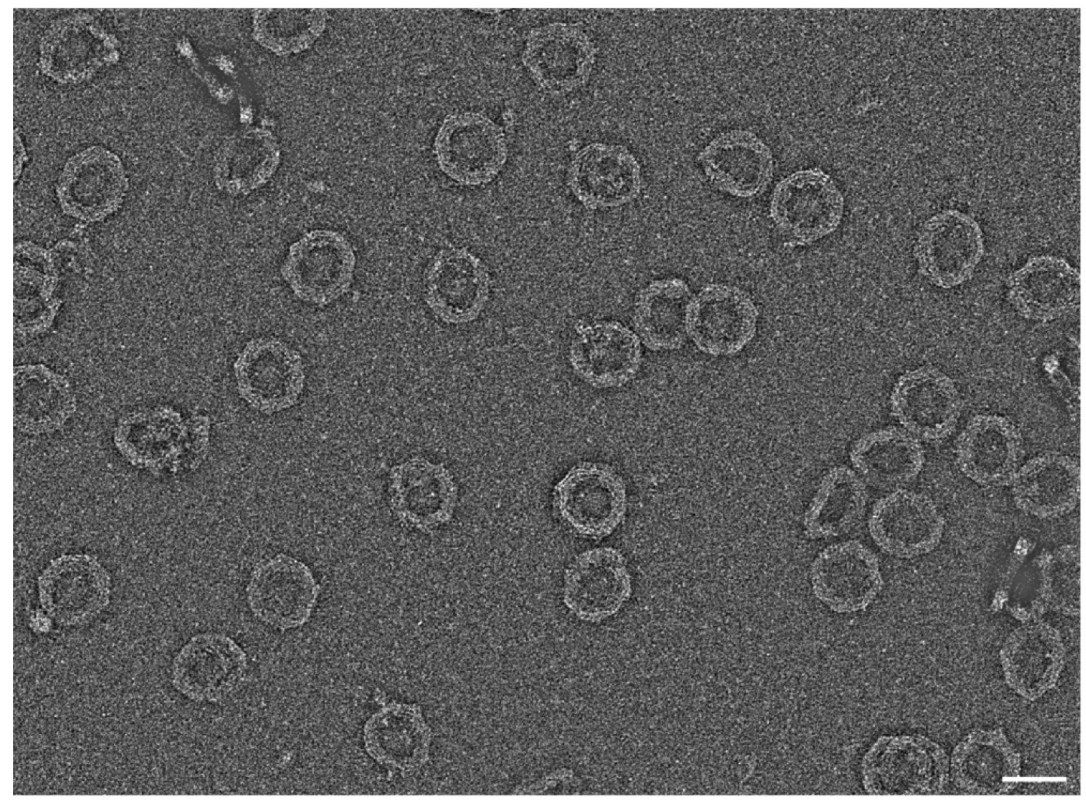

b
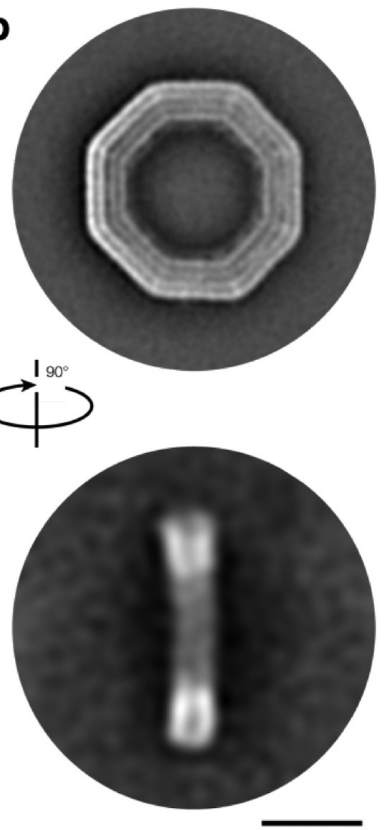

C
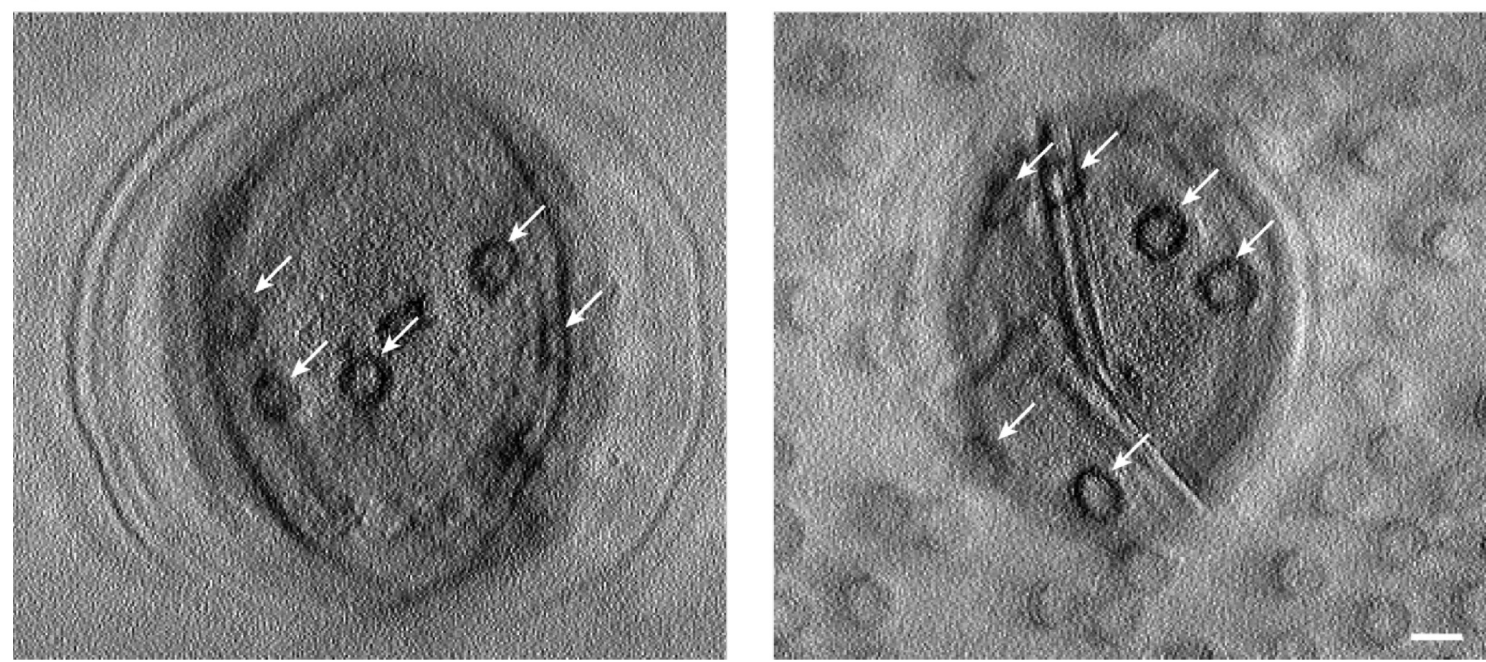

Figure 2. Negative-staining TEM and class averaging of the DNA origami pores. (a) Typical field of view of a negative-stained DNA origami sample. The pores in this image were prepared as described in the Methods section. The image is high-pass filtered with a radius of $20 \mathrm{~nm}$ and autoleveled in Photoshop. Scale bar: $50 \mathrm{~nm}$. (b) Top: Front view class average of $N=682$ single particles (Figure S12, left). Bottom: Side view class average of $N=80$ single particles (Figure S12, right). Scale bar: $25 \mathrm{~nm}$. (c) Two exemplary slices of negative-stained TEM tomograms calculated from EM tilt series, showing octagons (indicated by white arrows) embedded in the membrane of liposomes at a $z$ height away from the TEM support surface. See Figure S23 for a $z$-stack of the TEM tomography data. Scale bar: $50 \mathrm{~nm}$.

Atto647N dyes for fluorescence imaging, one at each corner on the bottom side of the octagon and four more on both ends of the asymmetric feature (Figure 1a). We iteratively refined the octagon design using gel electrophoretic mobility analysis as the read-out in order to minimize the occurrence of higherorder aggregates and improve the folding quality of the octagon object (Figures S3-S10).

The origami pore was coated with $\mathrm{K}_{10}-\mathrm{PEG}_{5 \mathrm{~K}}$ molecules, which consist of 10 positively charged lysine amino acids $(\mathrm{K})$ linked to a short polyethylene glycol chain (PEG, $5 \mathrm{kDa}$ ) (Figure $1 \mathrm{~b}$ ) as described in Ponnuswamy et al. ${ }^{21}$ This coating serves two purposes: (i) it stabilizes the octagon in low-ionicstrength environments, where uncoated DNA origami structures would otherwise disassemble, and (ii) it prevents the cholesterol-modified origami pores from aggregating in bulk. Figure 1c shows a schematic illustration of the incorporation of origami pores into the lipid membrane of a liposome (left). The zoom-ins highlight the interaction of the cholesterol-modified origami pores with the lipid molecules.

Characterization of the proper folding and verification of the intended dimensions of the structure were performed using negative-stain transmission electron microscopy (TEM) imaging and subsequent class averaging of single particles (Figure 2). Figure 2a shows a high-pass-filtered image of a typical field of view with several octagon pores. The overall shape of the majority of the particles matches the intended design, without obvious defects, in which 8 straight edges and 8 corners form an octagonal ring-like structure with a $\sim 35 \mathrm{~nm}$ 


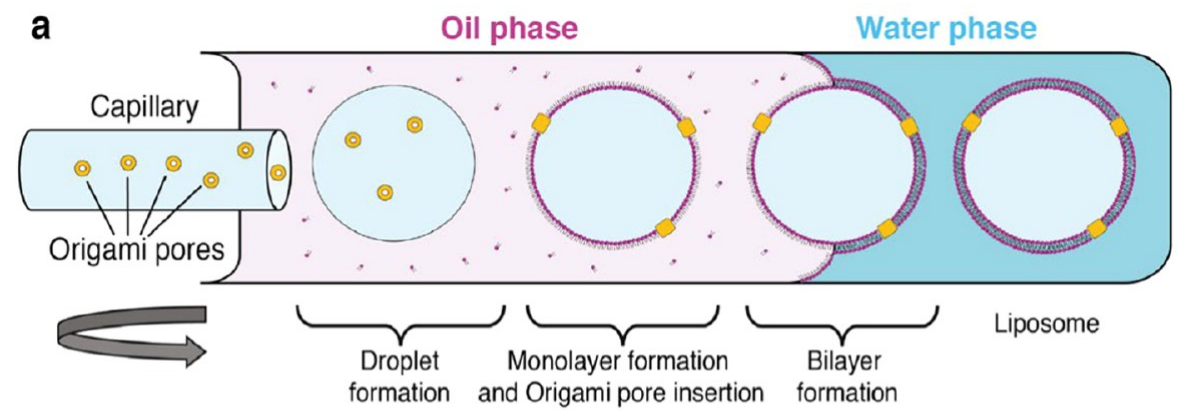

b

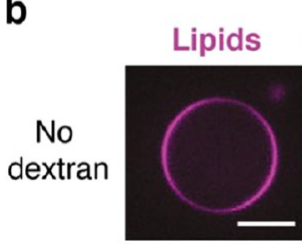

DNA origami

C

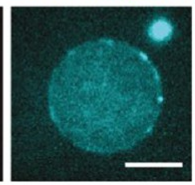

C Before bleaching
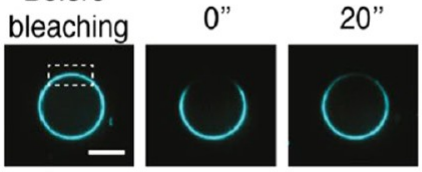
$180 "$

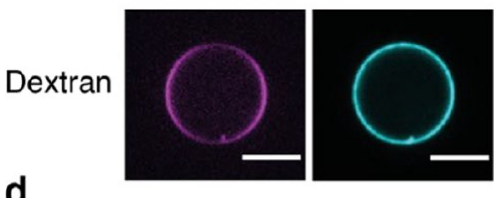

d
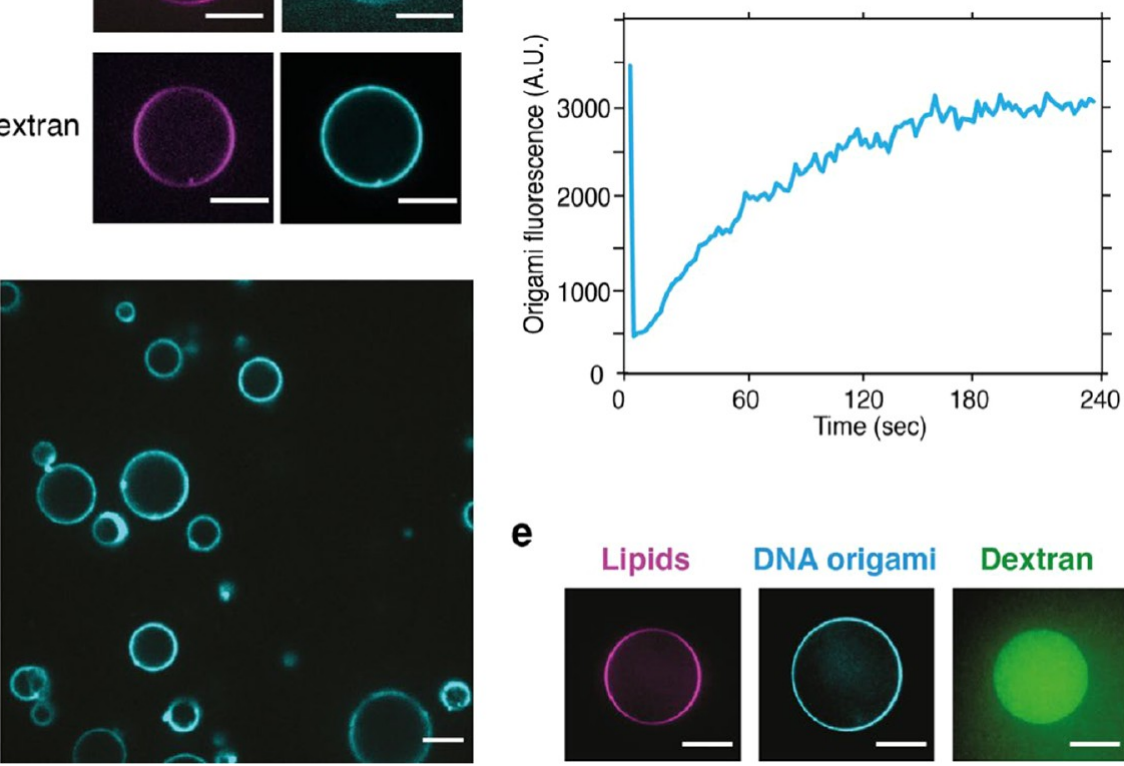

e

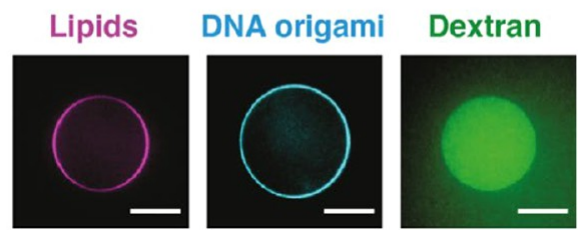

Figure 3. Reconstitution of wide DNA origami pores in GUVs by cDICE. (a) Schematics of the cDICE workflow. A section of the rotating chamber is depicted, including the oil and outer buffer phases. Origami pores are indicated in yellow. (b) Comparison of the efficiency of DNA origami pore localization at the membrane by addition of $2 \mathrm{MDa}$ dextran in the inner buffer. Without dextran, origami pores were observed to remain dispersed in bulk, whereas inclusion of dextran drove the origami pores to the lipid surface. (c) FRAP analysis of origami pores reconstituted in GUV that shows that the pores are mobile within the lipid bilayer. The dashed rectangle indicates the bleached area, which recovers within a few minutes. (d) Example of a large field of GUVs with reconstituted origami pores. (e) Example of a GUV retaining the $2 \mathrm{MDa}$ dextran in the lumen after $24 \mathrm{~h}$ incubation in buffer. Scale bars: $10 \mu \mathrm{m}$.

inner diameter and an $\sim 11 \mathrm{~nm}$ thickness. Most of the structures are oriented in a flat orientation on the surface, while a few can be seen in their side view.

We computed 2D class averages from single-particle micrographs (Figure S12), which resulted in two distinct images (Figure 2b), corresponding to front and side view transmission projections of the particles. The front view displays four distinct layers of double-stranded DNA. The asymmetric feature is visible on the outer two helices on the top side (Figure $2 \mathrm{~b}$, top). The class-averaged image of the side view shows less detail of the expected 4 layers of DNA and the asymmetric feature. This is likely due to the staining with uranyl formate and the tendency of the structures to adhere to the grid surface predominantly with their front side up, resulting in less side-view particles. From these images, we measured an inner diameter of $\sim 35 \mathrm{~nm}$ and a thickness of $\sim 11$ $\mathrm{nm}$ (Figure S13). Accounting for the $\mathrm{K}_{10}-\mathrm{PEG}_{5 \mathrm{~K}}$ coating by including a layer with a thickness of $2.4 \pm 1.3 \mathrm{~nm}$, as measured by Ponnuswamy et al., ${ }^{21}$ would result in an effective inner diameter of $\sim 30 \mathrm{~nm}$ and an effective height of $\sim 16 \mathrm{~nm}$ including the unstructured PEG brushes. Class-averaged images of $\mathrm{K}_{10}-\mathrm{PEG}_{5 \mathrm{~K}}$-coated and bare octagons match well (Figure S14), which shows that the coating preserves the global shape of the octagon pore.

Liposomes with octagons were imaged using negativestained EM tomography, showing that the octagons can be well incorporated within the lipid membrane (Movie 4 and Movie 5). The tomograms were calculated from the EM tilt series. Slices through the tomograms show octagons located at different heights on the surface of the liposomes (Figure S23). The top slices of the tomograms show octagons embedded in the lipid membrane on top of liposomes (Figure 2c). The global shape and the inner diameter of these octagons remained intact. 
a

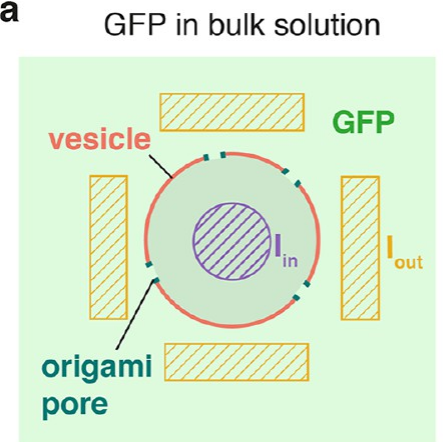

b - origami pores

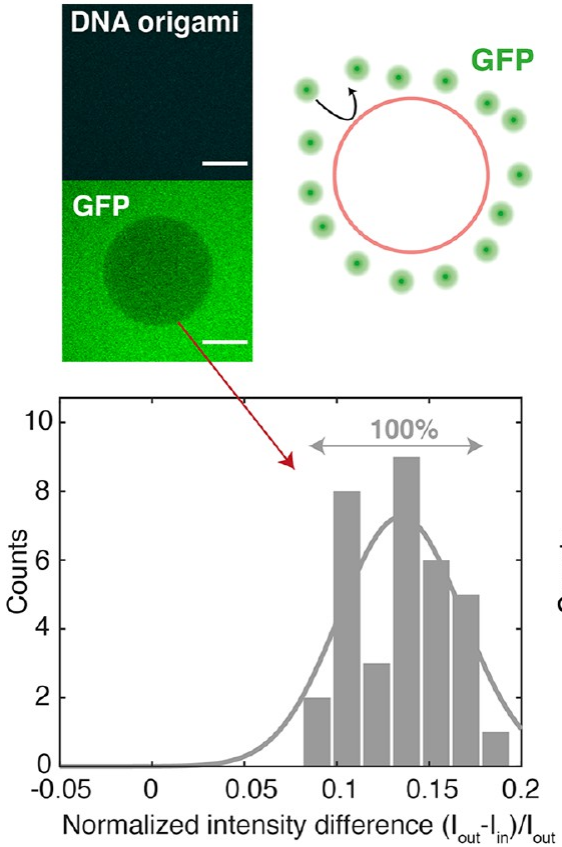

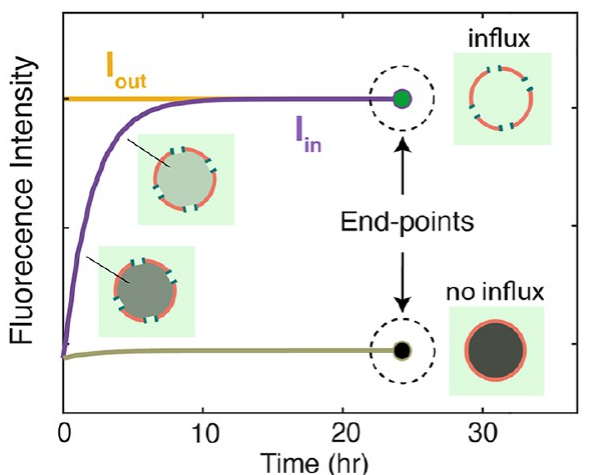

C

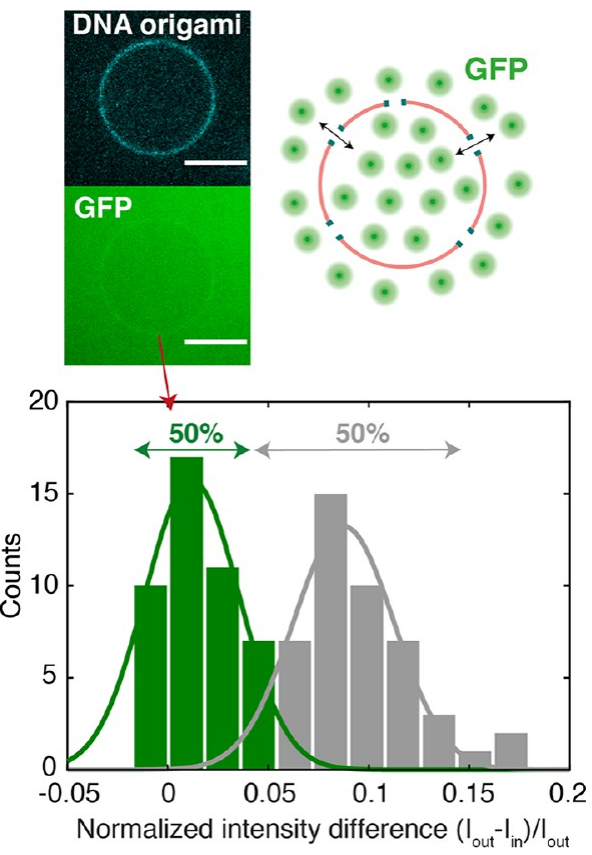

Figure 4. Test of GFP influx through origami pores. (a) Schematic of the influx experiment. Left: Sketch of a vesicle (red) containing pores (blue) that is incubated with GFP (green). Right: Fluorescence intensities $I_{\text {in }}$ and $I_{\text {out }}$ that are expected to be measured in a time-lapse experiment on the dashed purple and yellow areas, respectively. The right part of the graph illustrates the end-point intensities after $\sim 24 \mathrm{~h}$, after an initial transient. (b) Influx experiment with vesicles that were not subjected to DNA pore insertion. Top right: sketch of a poreless vesicle that excludes GFP. Top left: example of fluorescence image for a poreless vesicle (cyan) that prevents GFP (green) from entering the vesicle, showing up as a darker area signifying the absence of GFP. Bottom: Histogram of the normalized end-point intensity difference $\left(I_{\text {out }}\right.$ $\left.-I_{\text {in }}\right) / I_{\text {out }}$ for the poreless vesicles, verifying that no vesicles showed GFP influx. (c) Influx experiment with vesicles that were functionalized with origami pores. Top right: sketch of a pore-containing vesicle filled with GFP. Top left: example of fluorescence image for a porecontaining vesicle (cyan) that allows GFP to diffuse (green) into the vescicle, resulting in $I_{\text {out }}-I_{\text {in }} \approx 0$. Bottom: Histogram showing normalized end-point intensity difference $\left(I_{\text {out }}-I_{\text {in }}\right) / I_{\text {out }}$ for the poreless vesicles. We find that $\sim 50 \%$ of the vesicle population manifested GFP influx. Scale bars: $10 \mu \mathrm{m}$.

DNA Origami Pore Reconstitution in Liposomes by cDICE. We first tested spontaneous insertions observed for our $55 \mathrm{~nm}$ (outer diameter) large DNA origami pores into freestanding planar lipid bilayers or into preformed GUVs, under various conditions and with different nanopore versions with either 32 or 64 cholesterols. As expected, these attempts were unsuccessful. While membrane interactions drove the DNA origami objects to adhere on the GUVs (Figure S16), there was a total lack of influx of molecules (Figure S17), indicating that no actual pores were created with a functional transmembrane opening.

We therefore used an alternative approach where we incorporate the DNA origami pores during the process of the formation of the GUVs. We employed an inverted emulsion technique called $\mathrm{cDICE},{ }^{18}$ which yields unilamellar liposomes with good encapsulation of a large variety of macromolecules. ${ }^{22}$ Briefly, in cDICE, layers of buffer solution and lipid-in-oil suspension are deposited subsequently in a rotating chamber. As illustrated in Figure 3a, an inner buffer solution containing the origami pores is then injected through a capillary into the rotating lipid-in-oil suspension at a constant flow rate. Shear forces due to the rotation result in detachment of droplets from the capillary orifice. While traveling through the oil phase, these droplets acquire a first monolayer of lipids where the origami pores can insert. A second monolayer is subsequently formed when the droplets cross the oil-water 
a

1) Equilibrium state

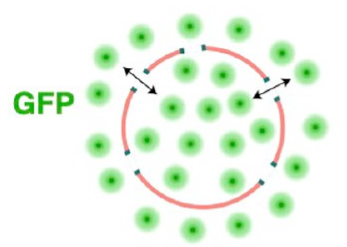

b
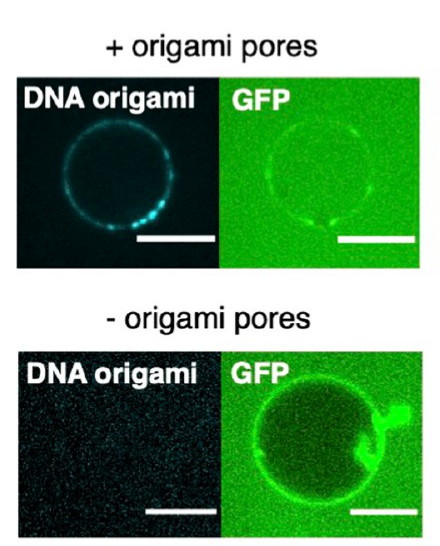

c

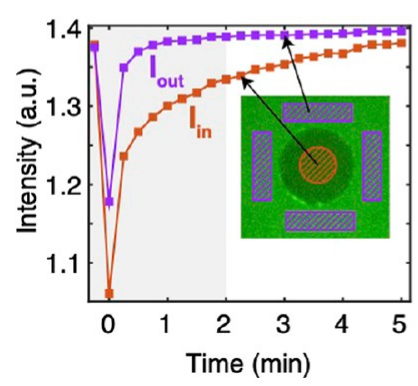

2) Photobleaching

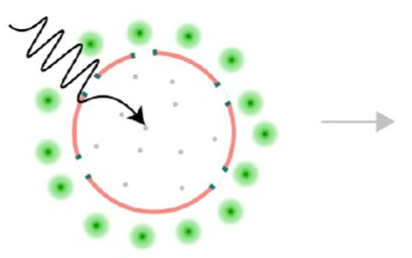

3) Recovery

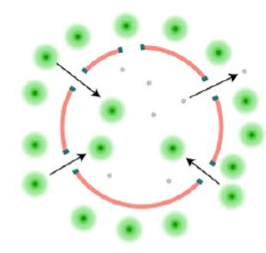

Full recovery after photobleaching

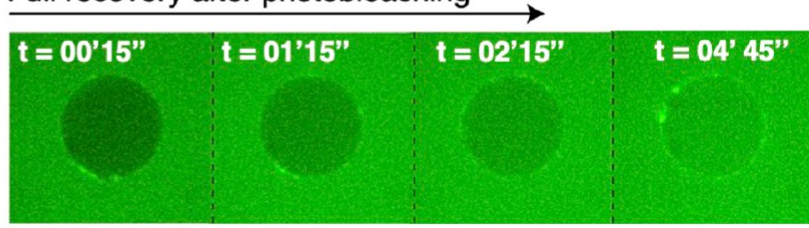

No recovery after photobleaching

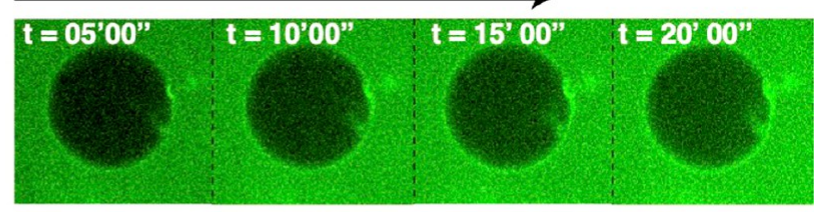

d

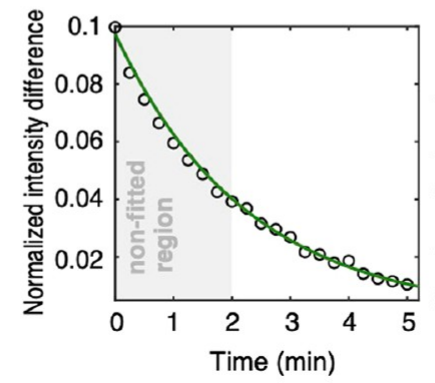

e

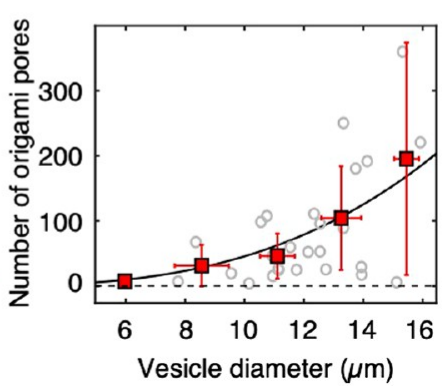

Figure 5. FRAP assay, modeling, and extraction of number of functional pores. (a) Schematic of the FRAP assay: (1) the vesicle is left to equilibrate with bulk solution containing GFP; (2) GFP molecules inside the vesicle are photobleached; (3) new GFP molecules are driven through the ultrawide origami pores from the outer solution, causing a recovery of the fluorescence signal. (b) Example of a FRAP experiment. Top left: image showing the pore-containing vesicle (origami pores in cyan) at equilibrium with the outer GFP (green) solution. Top right: series of frames showing recovery (within minutes) of the GFP signal after photobleaching. Bottom left: image showing a poreless vesicle (cyan) that excludes GFP (green) present in the outer solution. Bottom right: series of frames showing the absence of a signal increase after photobleaching. (c) Fluorescence intensities $I_{\text {in }}$ (red) and $I_{\text {out }}$ (purple) that reduce and recover after photobleaching for the vesicle shown in (b). Gray area denotes the initial transient, after which $I_{\text {out }}$ can be considered constant. Inset: Highlight of the regions where $I_{\text {in }}$ (red) and $I_{\text {out }}$ (purple) were measured. (d) Normalized intensity difference $I_{\mathrm{n} \text {,diff }}$ over time for the vesicle illustrated in (b). Data are fitted by eq 4 . Gray area was excluded from the fit as the model assumes a constant $I_{\text {out }}$. For such a $13.3 \mu$ m diameter vesicle, we estimated a number of functional pores $N_{\mathrm{p}}=250$. (e) Number of origami pores extracted from fits for different vesicles, as a function of vesicle diameter (gray circles). Averaged data over $2.5 \mu \mathrm{m}$ bins (red squares, error bars are standard deviation) show an increase of the number of pores as a function of vesicle diameter. Black line represents a cubic fit to the data. Scale bars: $10 \mu \mathrm{m}$.

interface, resulting in GUVs that are eventually collected from the outer buffer solution. In this CDICE process, we thus aimed to reconstitute the pore within the lipid monolayer that is formed in the water-in-oil droplets during the first step of cDICE. Whereas the origami rings with 64 cholesterols mainly resulted in formation of large aggregates at the droplet interface (Figure S18), the rings with 32 cholesterols appeared to interact with the lipid monolayer without inducing significant aggregation, suggesting a more promising route. Hereafter, we report the results for the 32 cholesterol version of the DNA origami rings.

In a standard cDICE protocol, ${ }^{22}$ the inner buffer solution that is injected contains either sucrose or Optiprep ${ }^{22}$ to obtain an optimal density of the inner GUV solution. We found, however, that in these conditions the reconstitution of 32 cholesterol origami rings was inefficient, with most of the origami pores remaining in solution after vesicle formation (Figure 3b). When we included high molecular weight dextran ( $\sim 2 \mathrm{MDa}$ ) in the inner buffer, we found that 32 cholesterol origami rings could be fully localized at the membrane (Figure $3 \mathrm{~b})$. Note that the choice of such a large dextran molecule (2 $\mathrm{MDa}$ ) was also motivated by the high diameter of gyration ( $~ 76 \mathrm{~nm}$; ref 23 ), which makes it large enough to stay in the vesicle despite the presence of the $30 \mathrm{~nm}$ wide DNA origami pores. 
The origami pores appeared to be evenly distributed on the GUV surface, only occasionally forming clusters, which then were also enriched in lipids (Movie 1 and Movie 2: $z$-stack and $3 \mathrm{D}$ rendering). Moreover, fluorescence recovery after photobleaching (FRAP) analysis revealed that DNA origami pores could freely diffuse within lipid bilayer of the vesicle membrane (Figure 3c). Hundreds of GUVs could be obtained from a single preparation (Figure 3d). The resulting GUVs retained the $2 \mathrm{MDa}$ dextran over prolonged periods of time ( $>24 \mathrm{~h}$, Figure $3 \mathrm{e}$ ) and were remarkably stable, remaining spherical in spite of osmotic changes, suggesting that the origami pores were very well able to equilibrate osmotic differences by allowing osmolytes to rapidly cross the membrane. All in all, the data indicate the successful insertion of wide DNA origami pores in the membrane of GUVs.

Protein Influx through the Pores Demonstrates Their Transmembrane Transport Capabilities. To assess whether the DNA origami pores were functional, that is, inserted in the intended orientation that allows for transmembrane transport through the central cavity of the octagon, we tested the influx of IBB-mEGFP-Cys, a $34.6 \mathrm{kDa}$ variant of the soluble green fluorescent protein that includes an importin $\beta$-binding domain ${ }^{24}$ (see Supporting Information S22), henceforth referred to as "GFP" for simplicity. We measured the normalized fluorescence intensity difference $I_{\text {n,diff }}=\left(I_{\text {out }}-\right.$ $\left.I_{\text {in }}\right) / I_{\text {out }}$ of the vesicles, that is, the difference between in-vesicle intensity $\left(I_{\text {in }}\right)$ and the outer bulk $\left(I_{\text {out }}\right)$ divided by $I_{\text {out }}$ (Figure 4a) after overnight incubation of the GUVs in an outer solution with $2.3 \mu \mathrm{M}$ of GFP. While poreless vesicles (control, Figure 4b) remained empty, yielding a finite value $I_{\mathrm{n}, \mathrm{diff}}=0.13$ \pm 0.05 (errors are standard deviation, $N=34$ ), the porecontaining liposomes showed an influx of GFP for about $50 \%$ of the vesicles (Figure 4c): half of the vesicles exhibited an almost complete saturation to $I_{\mathrm{n} 1 \text {,diff }}=0.01 \pm 0.03(N=45)$, while the other half yielded $I_{\mathrm{n} 2 \text {,diff }}=0.09 \pm 0.03(N=45)$, that is, no significant change compared to the control. We note that heterogeneity in GUV samples is a widely reported phenomenon $^{25}$ that apparently is associated with variations in the efficiency of pore insertion during vesicle formation in cDICE. Most importantly, a clear fraction of the liposomes unambiguously showed transmembrane transport of the folded proteins, indicating transport through the ultrawide DNA origami pores.

Next, we characterized the GFP influx rate quantitatively using a FRAP assay (Figure 5a). In this experiment, we selected pore-containing vesicles that showed that influx occurred during the overnight incubation (i.e., those with $I_{\mathrm{n} \text {,diff }}$ $\approx 0$ ), then photobleached the inner part, and subsequently measured the recovery of the fluorescence signal. An example of the FRAP experiment is shown in Figure 5b (top) and Movie 3. Starting from an equilibrium state where $I_{\text {in }} \approx I_{\text {out }}$ (Figure 5b, left), the vesicle fluorescence drops upon photobleaching to a level comparable to the one of an empty vesicle, to subsequently fully recover within a few minutes (Figure 5b, right). Notably, this time scale is much faster compared to those of previously reported fluorescence recovery with DNA origami pores. ${ }^{7,8}$ A control with vesicles lacking pores (Figure 5b, bottom) resulted in no appreciable recovery upon photobleaching. Figure 5c displays $I_{\text {in }}$ and $I_{\text {out }}$ for a pore-containing GUV as a function of time where, after initial transient behavior (gray area), $I_{\text {out }}$ is constant, whereas $I_{\text {in }}$ continues to slowly increase over time, which represents the influx of GFP through the ultrawide DNA origami pores.
To obtain the number of functional pores, we model the influx rate using a diffusion model derived from Fick's first law: ${ }^{26}$

$$
\phi=-D \frac{\mathrm{d} c}{\mathrm{~d} x}
$$

which describes the flux $\phi$ of GFP molecules with diffusion constant $D$, as a result of the concentration gradient $\mathrm{d} c / \mathrm{d} x$ between the outer $\left(c_{\text {out }}\right)$ and inner $\left(c_{\text {in }}\right)$ environment of the GUV. The flux $\phi$ is defined as number of molecules $d N$ crossing a membrane area $\mathrm{d} A$ in a time $\mathrm{d} t$. Since the vesicle volume $V$ is constant over time, the in-vesicle concentration $c_{\text {in }}(t)$ is a time-dependent variable that reflects the fact that the vesicle is filling up over time: $\frac{\mathrm{d} c_{\text {in }}(t)}{\mathrm{d} t}=\frac{1}{V} \frac{\mathrm{d} N}{\mathrm{~d} t}$. The outside concentration $c_{\text {out }}$ instead is assumed to be constant over time, which is reasonable given the large volume $(\sim 200 \mu \mathrm{L})$ of the bulk solution as compared to the $\sim$ picoliter volume of the vesicle. Assuming that the diffusion occurs through a number $N_{\mathrm{p}}$ of DNA origami pores that each have a length $L_{\mathrm{p}}$ and an area $A_{\mathrm{p}}$, yielding a total integrated area $A=A_{\mathrm{p}} N_{\mathrm{p}}$, we can rewrite eq 1 as the first-order differential equation

$$
\frac{\mathrm{d} c_{\text {in }}(t)}{\mathrm{d} t}=\frac{A D}{V} \frac{c_{\text {out }}-c_{\text {in }}(t)}{L_{\mathrm{p}}}
$$

which, with $c_{\text {in }}(t=0)=c_{\text {in, start }}$ has a solution

$$
c_{\text {in }}(t)=c_{\text {out }}-\left(c_{\text {out }}-c_{\text {in }, \text { start }}\right) e^{-\left(D_{\text {eff }} A / V L_{\mathrm{p}}\right) t}
$$

Note, however, that for the transport through the pores, we need to employ an effective diffusion constant $D_{\text {eff }}$ which is reduced compared to the bulk diffusion constant $D$ to account for the confined transport through the pores, as reported by Dechadilok and Deen. ${ }^{27}$ For our pore dimensions, this results in almost a factor of 2 decrease of the GFP diffusivity, that is, $D_{\text {eff }}=0.54 D_{\text {bulk. }}$. Upon rearranging eq 3 , we then finally obtain

$$
\frac{c_{\text {out }}-c_{\text {in }}(t)}{c_{\text {out }}}=\left(1-\frac{c_{\text {in }, \text { start }}}{c_{\text {out }}}\right) e^{-\left(D_{\text {eff }} A_{\mathrm{p}} N_{\mathrm{p}} / V L_{\mathrm{p}}\right) t}
$$

As the measured fluorescence intensities are a good measure for the protein concentrations of the fluorescent GFP, we can use eq 4 to fit the $I_{\text {n,diff }}(t)$ data, with $c_{\text {in,start }} / c_{\text {out }}$ and $N_{\mathrm{p}}$ as fit parameters. Figure $5 \mathrm{~d}$ provides an example, showing an excellent fit to the FRAP data. From fitting FRAP curves acquired from different vesicles, we find that the estimated number of pores $N_{\mathrm{p}}$ varies between a few to a few hundred per GUV. Despite the substantial scatter in the extracted $N_{\mathrm{p}}$ (gray circles in Figure $5 \mathrm{e}$ ), which we attribute to the stochasticity inherent to the vesicle production and origami insertion during cDICE, we do observe an overall increase of $N_{\mathrm{p}}$ with the vesicle diameter. Assuming that all origami pores would move from the volume into the membrane during GUV formation, $N_{\mathrm{p}}$ should scale with the cube of the vesicle diameter, which is consistent with the behavior observed (black line in Figure 5e).

Ultrawide DNA Origami Pores Act As a Molecular Sieve with $\sim 30 \mathrm{~nm}$ Cutoff. To provide evidence that our DNA origami pores indeed form open channels with an effective size of the order of the inner pore diameter of $30 \mathrm{~nm}$, we tested the influx of dextran-FITC molecules with a variety of sizes, viz., $D_{\mathrm{g}} \approx 15 \mathrm{~nm}(70 \mathrm{kDa}), 22 \mathrm{~nm}(150 \mathrm{kDa}), 28 \mathrm{~nm}$ $(250 \mathrm{kDa}), 39 \mathrm{~nm}(500 \mathrm{kDa})$, and $76 \mathrm{~nm}(2 \mathrm{MDa})$ (see Figure $6 \mathrm{a})$, where $D_{\mathrm{g}}$ is the diameter of gyration as measured by 
a

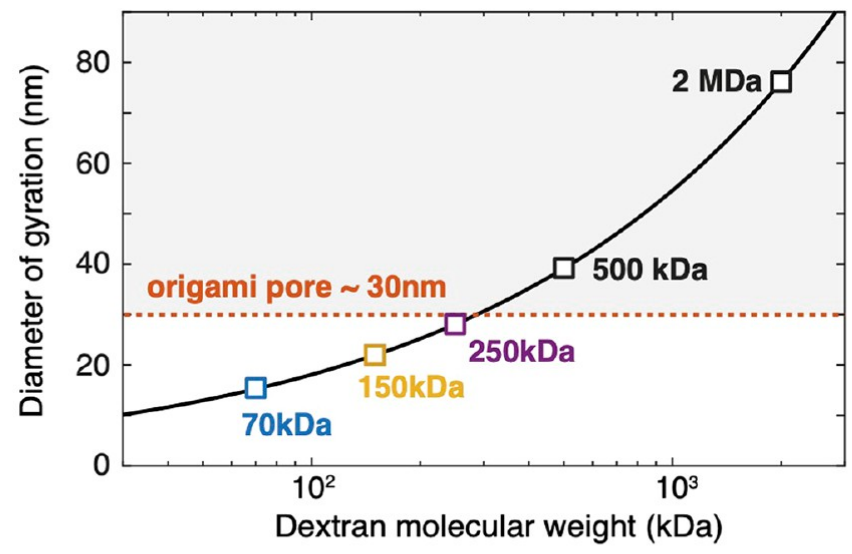

b

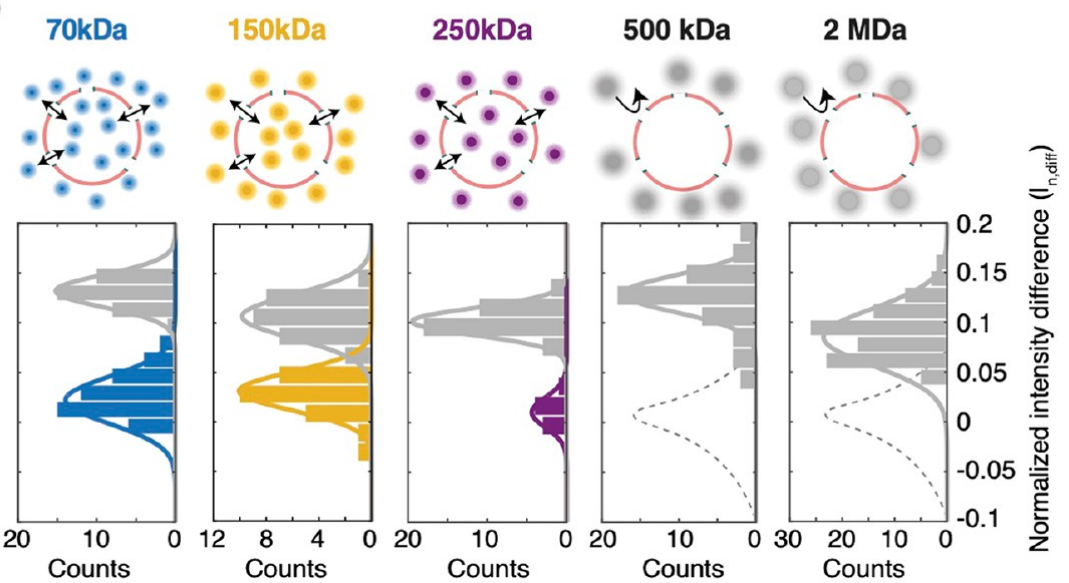

Figure 6. Size selectivity of DNA origami nanopores. (a) Diameter of gyration of dextran $D_{\mathrm{g}} v s$ molecular weight $M_{\mathrm{w}}$. Black line is a fit of $D_{\mathrm{g}}=$ $0.072 \times M_{\mathrm{w}}{ }^{0.48}$ (ref 23). Dextran sizes of $70 \mathrm{kDa}, 150 \mathrm{kDa}, 250 \mathrm{kDa}, 500 \mathrm{kDa}$, and $2 \mathrm{MDa}$ employed in our study are highlighted in blue, yellow, magenta, and black, respectively. Gray area underlies dextran sizes larger than the origami pore (red) which are not expected to translocate. (b) Histograms showing normalized intensity difference of pore-containing vesicles after overnight incubation the dextran molecules. Blue indicate vesicles that showed influx of $70 \mathrm{kDa}$, yellow bars for $150 \mathrm{kDa}$, magenta bars for $250 \mathrm{kDa}$, whereas gray bars represent empty vesicles. Dashed lines represent hypothetical populations of vesicles that would have been present if influx of $500 \mathrm{kDa}$ or 2 MDa through the origami pores had occurred.

Hanselmann and Burchard. ${ }^{23}$ As in the GFP influx experiment, we incubated the pore-containing vesicles with $2 \mu \mathrm{M}$ of dextran-FITC and measured the normalized intensity difference $I_{n, \text { diff }}$ after $\sim 24 \mathrm{~h}$. This was done independently for each of the 5 molecules, keeping the same molar concentration of dextran-FITC molecules in bulk solution. Similar to the GFP experiments (Figure $4 \mathrm{c}$ ), $I_{\mathrm{n} \text {,diff }}$ of pore-containing vesicles yielded two populations when incubated with $70 \mathrm{kDa}$ dextranFITC (Figure 6b, left), where 58\% manifested influx $\left(I_{\mathrm{n} 1, \text { diff,70k }}\right.$ $=0.02 \pm 0.03$, errors are standard deviation, $N=47$ ), while $42 \%$ remained empty $\left(I_{\mathrm{n} 2 \text {,diff,70k }}=0.13 \pm 0.02, N=34\right)$. Incubation with $150 \mathrm{kDa}$ dextran-FITC (Figure 6b, center-left) led to a similar result, with $47 \%$ GUVs that were filled $\left(I_{\mathrm{n} 1, \mathrm{diff}, 150 \mathrm{k}}=0.03 \pm 0.03, N=24\right)$ against $53 \%$ of empty vesicles $\left(I_{\mathrm{n} 2, \text { diff, } 150 \mathrm{k}}=0.11 \pm 0.03, N=27\right)$. Influx of the larger $250 \mathrm{kDa}$ dextran-FITC (Figure 6b, center) resulted in a reduced (19\%), yet present, population of filled vesicles $\left(I_{\mathrm{n} 1, \text { diff,250k }}=0.01 \pm 0.02, N=8\right)$, while $79 \%\left(I_{\mathrm{n} 2, \text { diff, } 250 \mathrm{k}}=0.10\right.$ $\pm 0.02, N=34$ ) showed no sign of influx. Importantly, this demonstrates that very large $(250 \mathrm{kDa} ; 28 \mathrm{~nm})$ macromolecules can be transported across the ultrawide DNA origami nanopores. By contrast, incubation with the even larger $500 \mathrm{kDa}$ and $2 \mathrm{MDa}$ dextran-FITC (Figure 6b, centerright and right) resulted in only one population of empty vesicles, with $I_{\mathrm{n}, \mathrm{diff}, 500 \mathrm{k}}=0.13 \pm 0.03(N=49)$ and $I_{\mathrm{n}, \mathrm{diff}, 2 \mathrm{M}}=$ $0.09 \pm 0.04(N=98)$, respectively, indicating a lack of any transmembrane transport during $24 \mathrm{~h}$. This is fully consistent with expectations as their size clearly exceeds the $30 \mathrm{~nm}$ size of the origami pore. It is also consistent with the use of $2 \mathrm{MDa}$ dextran as a macromolecular crowder (cf. Figure $3 \mathrm{e}$ ).

\section{CONCLUSIONS}

In this work, we achieved functional reconstitution of ultrawide DNA origami pores in giant liposomes, obtaining stable transmembrane pores of $30 \mathrm{~nm}$ inner diameter within the lipid bilayer. We successfully achieved the pore reconstitution by inserting the origami pore into a lipid monolayer followed by bilayer formation. This methodology lowers the energy barrier for pore insertion, allowing functional reconstitution of pores with much larger diameter than previously possible.

Our results show great potential for a number of biomimetic applications. For example, future efforts can be directed toward the functionalization of the origami pore with FG-nucleoporins from the nuclear pore complex, the macromolecular complex mediating transport across the nuclear envelope in eukaryotes, ${ }^{28}$ as reported in previous works. ${ }^{14,15}$ This will enable the creation of selective biomimetic pores that permit the exclusive transport of specific transport receptor proteins. While such 
biomimetic pores constitute an exciting platform for investigating nucleocytoplasmic transport in vitro, they may also be employed as a building block for synthetic cells, thus contributing to the in vitro reconstitution of an artificial model of a nucleus. This will, for example, aid the study of emergent properties of liposome-confined genomes ${ }^{29}$ by allowing the controlled injection of genome-processing proteins. Indeed, our DNA origami pores may be a great asset for building a synthetic cell as they provide a stable portal that is large enough for biological macromolecules like proteins and RNAs to enter a liposome. The long-term stability and functionality of these liposomes is a particularly useful feature in such synthetic biology applications. Finally, responsive drug delivery systems that release clinically relevant macromolecules such as antibodies upon stimulation by specific biomolecules could be envisioned, as well. We expect that the ultrawide DNA origami nanopores may thus find a broad variety of different applications.

\section{METHODS}

Design of Scaffolded DNA Origami Objects. The objects were designed using caDNAno v0.2. ${ }^{30}$

Optimal Folding Conditions of the Octagon. The folding reaction mixture for the octagon contained scaffold $\mathrm{DNA}^{31}$ at a final concentration of $10 \mathrm{nM}$ and oligonucleotide strands (IDT Integrated DNA Technologies) at $300 \mathrm{nM}$ each. The folding reaction buffer contained $5 \mathrm{mM}$ Tris, $1 \mathrm{mM}$ EDTA, $5 \mathrm{mM} \mathrm{NaCl}(\mathrm{pH}$ 8), and $24 \mathrm{mM}$ $\mathrm{MgCl}_{2}$. The folding reaction mixtures were subjected to the following thermal annealing ramp using TETRAD (MJ Research, now Biorad) thermal cycling devices: $15 \mathrm{~min}$ at $65^{\circ} \mathrm{C}$, followed by $16 \mathrm{~h}$ at $50^{\circ} \mathrm{C}$, followed by incubation at $20{ }^{\circ} \mathrm{C}$ before further sample preparation steps.

Purification of DNA Origami Nanostructures. Excess oligonucleotides were removed via ultrafiltration (Amicon Ultra 0.5 $\mathrm{mL}$ Ultracel filters, 50K) with FoB5 (5 mM Tris, 1 mM EDTA, $5 \mathrm{mM}$ $\mathrm{NaCl}$, and $5 \mathrm{mM} \mathrm{MgCl}_{2}$ ) buffer. ${ }^{19}$ All centrifugation steps were performed at $10 \mathrm{kG}$ for $3 \mathrm{~min}$ at $25^{\circ} \mathrm{C}$. Step 1: $0.5 \mathrm{~mL}$ of FoB5 buffer was added to the filters and centrifuged. Step 2: $0.1 \mathrm{~mL}$ of folded object sample and $0.4 \mathrm{~mL}$ of FoB5 buffer were added to the filters and centrifuged. Step 3: $0.45 \mathrm{~mL}$ of FoB5 buffer was added to the filters and centrifuged. Step 3 was repeated 3 times before a final retrieving step: the filter insets were turned upside down, placed into a new tube, and centrifuged. This concludes one round of ultrafiltration.

Coating of the Octagon. Different volumes of purified octagons were mixed with PEG-oligolysine (K10-PEG5k). The optimal amount of $\mathrm{K}_{10}-\mathrm{PEG}_{5 \mathrm{~K}}$ added was determined based on the ratio of nitrogen in amines to the phosphates in DNA (Ponnuswamy et al. ${ }^{21}$ ) to a final ratio of $0.75: 1(\mathrm{~N} / \mathrm{P})$.

Negative-Stain TEM. A carbon Formvar grid (Electron Microscopy Sciences) was plasma-treated (45 s, $35 \mathrm{~mA}$ ) right before use. Five microliters of uncoated octagon sample (10-50 nM DNA object concentrations, 5-20 mM MgCl 2 concentration) was pipetted onto the grid. Coated octagon samples were pipetted onto the grids without plasma treatment in order to reduce positive staining effects. The sample droplets were incubated for $30-120 \mathrm{~s}$ on the grids and then blotted away with filter paper. A $5 \mu \mathrm{L}$ droplet of $2 \%$ aqueous uranyl formate (UFO) solution containing $25 \mathrm{mM}$ sodium hydroxide was pipetted onto the grid and immediately blotted away. A $20 \mu \mathrm{L}$ UFO droplet was then pipetted onto the grid, incubated for $30 \mathrm{~s}$, and blotted away. The grids were then air-dried for $20 \mathrm{~min}$ before imaging on a Philips CM100 and an FEI Tecnai 120 microscope. Particles were picked automatically using crYOLO $^{32}$ and subsequently class averaged using Relion3.0.33

Negative-stain EM tomography was used to visualize octagons at the surface of liposomes. The samples $(5 \mu \mathrm{L})$ containing liposomes with incorporated octagons were incubated on the grid surface until the liquid evaporated $(\sim 15 \mathrm{~min})$. The sample was not blotted away using filter paper in an effort not to damage the liposomes through shear forces. All other grid preparation steps were the same as described before.

The tilt series were acquired at a magnification of $30000 \times$ using an FEI Tecnai 120. The stage was tilted from $-50^{\circ}$ to $+50^{\circ}$, and micrographs were acquired every $2^{\circ}$. The tilt series were then processed using Etomo (IMOD) ${ }^{34}$ to acquire tomograms. The micrographs were first aligned by calculating a cross-correlation of the consecutive images. The tomograms were then generated using a filtered back projection. The Gaussian filter used a cutoff of 0.35 and a falloff of 0.035 .

Gel Electrophoresis of Octagon Samples. Octagon samples were electrophoresed on 1.5 and $2.0 \%$ agarose gels containing $0.5 \times$ Tris-borate-EDTA and $5 \mathrm{mM} \mathrm{MgCl} 2$ (unless noted otherwise) for around $1-2 \mathrm{~h}$ at 70 or $90 \mathrm{~V}$ bias voltage in a gel box, cooled in a water or ice water bath. The loaded samples contained final monomer concentrations of $10 \mathrm{nM}$ (unless otherwise noted). The gels were scanned with a Typhoon FLA 9500 laser scanner (GE Healthcare) at a resolution of 25 or $50 \mu \mathrm{m} /$ pixel. Further enhancement of the resulting images or parts of images was performed using Photoshop CS5.

\section{DNA Origami Sample Preparation Protocol.}

1. Folding of DNA origami samples at previously described optimal folding conditions.

2. Three rounds of ultrafiltration to purify the samples of excess oligonucleotides.

3. Addition of Atto647N modified oligonucleotide at a final oligonucleotide/DNA origami ratio of 48:1 (12 handles per DNA origami; effective 4:1 ratio).

4. One round of ultrafiltration to purify the samples of excess Atto647N oligonucleotides.

5. Addition of PEG-oligolysine at $\mathrm{N} / \mathrm{P}$ ratio of $0.75: 1$, incubation at room temperature overnight.

6. Addition of cholesterol modified oligonucleotide at a final oligonucleotide/DNA origami ratio of 128:1 (32 handles per DNA origami; effective 4:1 ratio).

7. One round of ultrafiltration to purify the samples of excess cholesterol oligonucleotides.

Reagents. The $2 \mathrm{MDa}$ dextran (D5376), $\mathrm{MgCl}_{2}$ (M8266), glucose (G7021), Optiprep (60\% (w/v) iodixanol in water, D1556), silicone oil (317667), and mineral oil (M3516-1L) were purchased from Sigma-Aldrich. Tris-HCl (10812846001) was purchased from Roche. DOPC (1,2-dioleoyl-sn-glycero-3-phosphocholine) (850375) and DOPE-Rhodamine (1,2-dioleoyl-sn-glycero-3-phosphoethanolamine- $N$-(lissamine rhodamine B sulfonyl) (ammonium salt)) (810150C) were purchased from Avanti Lipids. Lipids were stored and resuspended in anhydrous chloroform (288306, Sigma Alrich). Sodium dodecyl sulfate employed for cleaning steps was purchased from Sigma-Aldrich. UltraPure bovine serum albumin used for passivation of the glass coverslips was purchased by ThermoFisher. For the influx experiments, we employed $70 \mathrm{kDa}$ dextran-FITC $($ FITC/glucose $(\mathrm{mol} / \mathrm{mol})=0.004)$ (Sigma-Aldrich), $150 \mathrm{kDa}$ dextran-FITC $($ FITC/glucose $(\mathrm{mol} / \mathrm{mol})=0.004)($ Sigma-Aldrich $)$, $250 \mathrm{kDa}$ dextran-FITC $($ FITC $/$ glucose $(\mathrm{mol} / \mathrm{mol})=0.003-0.020)$ (Sigma-Aldrich), $500 \mathrm{kDa}$ dextran-FITC (FITC/glucose $(\mathrm{mol} / \mathrm{mol})$ $=0.003-0.020)$ (Sigma-Aldrich), and $2 \mathrm{MDa}$ dextran-FITC (FITC/ glucose $(\mathrm{mol} / \mathrm{mol})=0.001-0.004)$ (Sigma-Aldrich) .

Expression and Purification of IBB-eGFP. For the expression of His14-TEV-IBB-mEGFP-Cys ("IBB-GFP"), plasmid pSF807 (Eisele et al. ${ }^{35}$ ) was transformed into Escherichia coli BLR cells. Cultures were grown in TB medium supplemented with $50 \mu \mathrm{g} / \mathrm{mL}$ kanamycin, and expression was induced with $0.5 \mathrm{mM}$ IPTG during overnight incubation at $18{ }^{\circ} \mathrm{C}$, with shaking at $150 \mathrm{rpm}$ in baffled flasks. Cells were harvested by centrifugation (10 min at $4000 \mathrm{rpm}$, JLA8.1000 rotor), washed in $1 \times$ phosphate-buffered saline, and lysed using a French Press (Constant Systems) at $20 \mathrm{kpsi}$ at $4{ }^{\circ} \mathrm{C}$ in buffer A $(2 \mathrm{M}$ $\mathrm{NaCl}, 50 \mathrm{mM}$ Tris/ $\mathrm{HCl} \mathrm{pH}$ 8.0, $5 \mathrm{mM} \mathrm{MgCl}_{2}$ ). Unbroken cells, debris, and aggregates were pelleted in a $\mathrm{Ti} 45$ rotor $(30 \mathrm{~min}, 40000$ $\mathrm{rpm}, 4{ }^{\circ} \mathrm{C}$ ), and the lysate resulting from $1 \mathrm{~L}$ cell culture was applied 
to $2 \mathrm{~mL}$ (bed volume) of Ni-NTA matrix prewashed with buffer A. After $60 \mathrm{~min}$ incubation while slowly inverting the tube in a cold room $\left(4{ }^{\circ} \mathrm{C}\right)$, the matrix was washed extensively with buffer $\mathrm{A}$ supplemented with $60 \mathrm{mM}$ imidazole and buffer B (50 mM Tris/ $\mathrm{HCl}$ $\mathrm{pH} 7.5,300 \mathrm{mM} \mathrm{NaCl}, 5 \mathrm{mM} \mathrm{MgCl}, 1 \mathrm{mM} \beta \mathrm{ME}, 10 \%$ glycerol). Following overnight elution by homemade TEV protease in buffer $\mathrm{B}$, the eluate was concentrated by ultracentrifugation and further purified by size exclusion chromatography on a Superdex 200 column (GE Healthcare) pre-equilibrated with buffer B supplemented with 0.5 $\mathrm{mM}$ EDTA. The final IBB-GFP sample with a concentration of 138 $\mu \mathrm{M}$ was aliquoted, snap frozen in liquid nitrogen, and stored at -80 ${ }^{\circ} \mathrm{C}$.

CDICE Buffers and Solutions. The encapsulated inner solution contains $50 \mathrm{mM}$ Tris- $\mathrm{HCl} \mathrm{pH} 7.4,5 \mathrm{mM} \mathrm{MgCl}, 15 \mu \mathrm{M} 2 \mathrm{MDa}$ dextran, $2 \mathrm{nM}$ DNA origami pores. The outer solution contains glucose in Milli-Q water and is osmotically matched to the inner solution. The oil phase is a mixture of silicone and mineral oil (4:1 ratio) and contains lipids dissolved at $0.2 \mathrm{mg} / \mathrm{mL}$. The lipid-in-oil suspension was prepared as in Van de Cauter et al. ${ }^{22}$ and used immediately.

Preparation of Lipid-in-Oil Suspension. DOPC and DOPERhodamine lipids solubilized in chloroform were mixed at 99.9:0.1 ratio and blow-dried with pure nitrogen. Inside a glovebox filled with pure nitrogen, lipids were subsequently resolubilized with anhydrous chloroform. The freshly prepared mixture of silicone and mineral oil was added to the lipids dropwise while vortexing at $1400 \mathrm{rpm}$. The lipid-in-oil solution was finally vortexed at $2800 \mathrm{rpm}$ for $2 \mathrm{~min}$ and further sonicated in an ice bath for $15 \mathrm{~min}$.

CDICE Equipment and GUV Production. The rotating component of the cDICE device is built from a magnetic stirrer L71 (LAbinco). A Teflon adaptor connects the rotating shaft with the cDICE chamber, assembled from a standard $3.5 \mathrm{~cm}$ cell culture dish (corning) modified to decrease the height. The chamber has an inner height of $8 \mathrm{~mm}$ and a circular opening of $10 \mathrm{~mm}$ on the top to allow insertion of the capillary and collection of the GUVs. The detailed protocol for GUV production is reported in Van de Cauter et al., ${ }^{22}$ while here we describe it briefly. First, the cDICE chamber is set to rotate at $300 \mathrm{rpm}$. Next, $350 \mu \mathrm{L}$ of outer aqueous solution is injected into the rotating the chamber, followed by $1 \mathrm{~mL}$ of lipid-in-oil solution, resulting in the two phases being stacked, as depicted in Figure 3a. The tip of a $100 \mu \mathrm{m}$ PEEK capillary tube (211633-3, BGB) is then inserted into the oil phase of the rotating chamber, allowing for a continuous supply of inner aqueous solution into the oil phase by a pressure pump (MFCS-EZ, Fluigent) set at 900 mbar. After 15 min, the rotating speed is set to zero, and GUVs are collected from the outer aqueous solution and moved to a prepassivated imaging well to carry out the fluorescence experiments.

Data Collection and Analysis. Fluorescence images were acquired using spinning disk confocal laser microscopy (Olympus IXB1/BX61 microscope, 60× objective, iXon camera) with Andor iQ3 software. To induce GFP photobleaching, we employed raster scanning with a $491 \mathrm{~nm}$ laser (at $9.8 \mathrm{~mW}$ ) over the region of interest. To measure the recovery signal, frames were collected every $15 \mathrm{~s}$, starting right after the photobleaching event. Fluorescence images were analyzed and processed using ImageJ (v2.1.0). The extracted fluorescence data were plotted and fitted using MATLAB.

Diffusion Model. Fluorescence recovery data were fitted with a diffusion model, described by eq 4, derived from Fick's law of diffusion. Whereas $c_{\text {in,start }} / c_{\text {out }}$ and $N_{\mathrm{p}}$ were free parameters of the fit, all the other parameters were known or could be calculated. More particularly, the area of the pore $A_{\mathrm{p}}=745.6 \mathrm{~nm}^{2}$ was calculated from assuming an octagonal pore with an effective radius of $15 \mathrm{~nm}$ (as discussed in the text). The effective diffusion constant $D_{\text {eff }}=47 \mu \mathrm{m}^{2} / \mathrm{s}$ of GFP was calculated using the formula provided by Dechadilok and Deen: ${ }^{27}$

$$
\begin{aligned}
& \frac{D_{\text {eff }}}{D_{\text {bulk }}}=1+\frac{9}{8}\left(\frac{R_{\mathrm{g}}}{R_{\mathrm{p}}}\right) \ln \left(\frac{R_{\mathrm{g}}}{R_{\mathrm{p}}}\right)-1.56034\left(\frac{R_{\mathrm{g}}}{R_{\mathrm{p}}}\right)+0.528155\left(\frac{R_{\mathrm{g}}}{R_{\mathrm{p}}}\right)^{2} \\
& +1.91521\left(\frac{R_{\mathrm{g}}}{R_{\mathrm{p}}}\right)^{3}-2.81903\left(\frac{R_{\mathrm{g}}}{R_{\mathrm{p}}}\right)^{4}+0.270788\left(\frac{R_{\mathrm{g}}}{R_{\mathrm{p}}}\right)^{5} \\
& +1.10115\left(\frac{R_{\mathrm{g}}}{R_{\mathrm{p}}}\right)^{6}-0.435933\left(\frac{R_{\mathrm{g}}}{R_{\mathrm{p}}}\right)^{7}
\end{aligned}
$$

where $R_{\mathrm{g}}$ is the molecule radius of gyration (for GFP, $R_{\mathrm{g}}=1.77 \mathrm{~nm}$ ), $R_{\mathrm{p}}$ is the pore radius (here $15 \mathrm{~nm}$ ), and $D_{\text {bulk }}$ is the diffusion constant of the molecule in free solution (for GFP, $D_{\text {bulk }}=87 \mu \mathrm{m}^{2} / \mathrm{s}$, ref 36 ). The channel length $L=16 \mathrm{~nm}$ was assumed to equal the effective height of the origami, which was measured by TEM imaging (Figure $\mathrm{S} 13 \mathrm{~b})$. Finally, $V$ was the volume of the vesicle, which was estimated from the fluorescence images.

\section{ASSOCIATED CONTENT}

\section{(s) Supporting Information}

The Supporting Information is available free of charge at https://pubs.acs.org/doi/10.1021/acsnano.1c01669.

Design, layout, sequence of the DNA origami pore; agarose gels for the screening protocol, TEM single particles used for class averaging, additional images on the EM tomography of the liposomes, fluorescence images showing results of control experiments and raw data used for fitting, captions describing Movies 1-5 (PDF)

Movies 1-5 (ZIP)

\section{AUTHOR INFORMATION}

\section{Corresponding Authors}

Cees Dekker - Department of Bionanoscience, Kavli Institute of Nanoscience, Delft University of Technology, $2629 \mathrm{HZ}$ Delft, The Netherlands; O orcid.org/0000-0001-6273071X; Email: c.dekker@tudelft.nl

Hendrik Dietz - Physik Department, Technische Universität München, Garching bei München D-85748, Germany; Email: dietz@tum.de

\section{Authors}

Alessio Fragasso - Department of Bionanoscience, Kavli Institute of Nanoscience, Delft University of Technology, 2629 HZ Delft, The Netherlands; orcid.org/0000-0001-83662998

Nicola De Franceschi - Department of Bionanoscience, Kavli Institute of Nanoscience, Delft University of Technology, 2629 HZ Delft, The Netherlands; (1) orcid.org/0000-0002-7221613X

Pierre Stömmer - Physik Department, Technische Universität München, Garching bei München D-85748, Germany; - orcid.org/0000-0003-1683-3392

Eli O. van der Sluis - Department of Bionanoscience, Kavli Institute of Nanoscience, Delft University of Technology, 2629 HZ Delft, The Netherlands

Complete contact information is available at: https://pubs.acs.org/10.1021/acsnano.1c01669

\section{Author Contributions}

A.F., H.D., and C.D. conceived the research. N.D.F. and C.D. set up cDICE and devised the reconstitution protocol. P.S. and H.D. devised the DNA origami nanopores. P.S. carried out 
assembly, quality control, and TEM imaging of the DNA origami nanopores. A.F. and N.D.F. carried out influx experiments. A.F. performed analysis and modeling of influx data. E.O.v.d.S. expressed and purified the GFP. A.F., N.D.F., P.S., H.D., and C.D. wrote the manuscript. A.F., N.D.F., and P.S. contributed equally to this work.

\section{Notes}

The authors declare no competing financial interest.

The manuscript has been previously deposited on a preprint server as Alessio Fragasso, Nicola De Franceschi, Pierre Stömmer, Eli O. van der Sluis, Hendrik Dietz, Cees Dekker. Reconstitution of Ultrawide DNA Origami Pores in Liposomes for Transmembrane Transport of Macromolecules. bioRxiv 2021, 432733; 10.1101/2021.02.24.432733 (accessed February 24,2021$)$.

\section{ACKNOWLEDGMENTS}

We thank Kristina Ganzinger and Gijsje Koenderink for helpful discussions and technical advice in setting up cDICE; Federico Fanalista, Nils Klughammer, and Jaco van der Torre for discussions; Jeremie Capoulade for technical support on confocal microscopy; and Dimitri de Roos for constructing the cDICE steup. This work was supported by a European Research Council Consolidator Grant to H.D. (GA No. 724261), the Deutsche Forschungsgemeinschaft through grants provided within the Gottfried-Wilhelm-Leibniz Program and the SFB863 Project ID 111166240 TPA9 (to H.D.) C.D. acknowledges funding support from the ERC Advanced Grant 883684, NWO grants 16PR3242-1, BBOL.737.016.016, and OCENW.GROOT.2019.068, and the NanoFront and BaSyC programs.

\section{REFERENCES}

(1) Rothemund, P. W. K. Folding DNA to Create Nanoscale Shapes and Patterns. Nature 2006, 440 (7082), 297-302.

(2) Douglas, S. M.; Dietz, H.; Liedl, T.; Högberg, B.; Graf, F.; Shih, W. M. Self-Assembly of DNA into Nanoscale Three-Dimensional Shapes. Nature 2009, 459 (7245), 414-418.

(3) Hernández-Ainsa, S.; Keyser, U. F. DNA Origami Nanopores: Developments, Challenges and Perspectives. Nanoscale 2014, 6 (23), 14121-14132.

(4) Sugawara, T.; Yamashita, D.; Kato, K.; Peng, Z.; Ueda, J.; Kaneko, J.; Kamio, Y.; Tanaka, Y.; Yao, M. Structural Basis for PoreForming Mechanism of Staphylococcal $\alpha$-Hemolysin. Toxicon 2015, 108, 226-231.

(5) Martin, T. G.; Simmel, F. C.; Arnaut, V.; Mayer, M.; Dietz, H.; Langecker, M.; Renner, S.; List, J. Synthetic Lipid Membrane Channels Formed by Designed DNA Nanostructures. Science (Washington, DC, U. S.) 2012, 338 (6109), 932-936.

(6) Gyenes, B.; Göpfrich, K.; Winterhalter, M.; Li, C.-Y.; Ohmann, A.; Yoo, J.; Aksimentiev, A.; Ricci, M.; Bhamidimarri, S. P.; Keyser, U. F. Large-Conductance Transmembrane Porin Made from DNA Origami. ACS Nano 2016, 10 (9), 8207-8214.

(7) Krishnan, S.; Ziegler, D.; Arnaut, V.; Martin, T. G.; Kapsner, K.; Henneberg, K.; Bausch, A. R.; Dietz, H.; Simmel, F. C. Molecular Transport through Large-Diameter DNA Nanopores. Nat. Commun. 2016, 7, 1-7.

(8) Thomsen, R. P.; Malle, M. G.; Okholm, A. H.; Krishnan, S.; Bohr, S. S.; Sørensen, R. S.; Ries, O.; Vogel, S.; Simmel, F. C.; Hatzakis, N. S.; Kjems, J. A Large Size-Selective DNA Nanopore with Sensing Applications. Nat. Commun. 2019, 10, 1-10.

(9) Burns, J. R.; Seifert, A.; Fertig, N.; Howorka, S. A Biomimetic DNA-Based Channel for the Ligand-Controlled Transport of Charged Molecular Cargo across a Biological Membrane. Nat. Nanotechnol. 2016, 11 (2), 152-156.
(10) Iwabuchi, S.; Kawamata, I.; Murata, S.; Nomura, S. M. Large, Square-Shaped, DNA Origami Nanopore with Sealing Function on Giant Vesicle Membrane. Chem. Commun. 2021, 57, 2990-2993.

(11) Ramezani, H.; Dietz, H. Building Machines with DNA Molecules. Nat. Rev. Genet. 2020, 21 (1), 5-26.

(12) Wagenbauer, K. F.; Sigl, C.; Dietz, H. Gigadalton-Scale ShapeProgrammable DNA Assemblies. Nature 2017, 552 (7683), 78-83.

(13) Ohmann, A.; Li, C. Y.; Maffeo, C.; Al Nahas, K.; Baumann, K. N.; Göpfrich, K.; Yoo, J.; Keyser, U. F.; Aksimentiev, A. A Synthetic Enzyme Built from DNA Flips 107 Lipids per Second in Biological Membranes. Nat. Commun. 2018, 9 (1), 1-9.

(14) Ketterer, P.; Ananth, A. N.; Laman Trip, D. S.; Mishra, A.; Bertosin, E.; Ganji, M.; Van Der Torre, J.; Onck, P.; Dietz, H.; Dekker, C. DNA Origami Scaffold for Studying Intrinsically Disordered Proteins of the Nuclear Pore Complex. Nat. Commun. 2018, 9 (1), 1-8.

(15) Fisher, P. D. E.; Shen, Q.; Akpinar, B.; Davis, L. K.; Chung, K. K. H.; Baddeley, D.; Sarić, A.; Melia, T. J.; Hoogenboom, B. W.; Lin, C.; Lusk, C. P. A Programmable DNA Origami Platform for Organizing Intrinsically Disordered Nucleoporins within Nanopore Confinement. ACS Nano 2018, 12 (2), 1508-1518.

(16) Exerowa, D.; Kashchiev, D.; Platikanov, D. Stability and Permeability of Amphiphile Bilayers. Adv. Colloid Interface Sci. 1992, 40 (C), 201-256.

(17) Akimov, S. A.; Volynsky, P. E.; Galimzyanov, T. R.; Kuzmin, P. I.; Pavlov, K. V.; Batishchev, O. V. Pore Formation in Lipid Membrane I: Continuous Reversible Trajectory from Intact Bilayer through Hydrophobic Defect to Transversal Pore. Sci. Rep. 2017, 7 (1), $1-20$.

(18) Abkarian, M.; Loiseau, E.; Massiera, G. Continuous Droplet Interface Crossing Encapsulation (CDICE) for High Throughput Monodisperse Vesicle Design. Soft Matter 2011, 7 (10), 4610-4614.

(19) Wagenbauer, K. F.; Engelhardt, F. A. S.; Stahl, E.; Hechtl, V. K.; Stömmer, P.; Seebacher, F.; Meregalli, L.; Ketterer, P.; Gerling, T.; Dietz, H. How We Make DNA Origami. ChemBioChem 2017, 18 (19), 1873-1885.

(20) Dietz, H.; Douglas, S. M.; Shih, W. M. Folding DNA into Twisted and Curved Nanoscale Shapes. Science (Washington, DC, U. S.) 2009, 325 (5941), 725-730.

(21) Ponnuswamy, N.; Bastings, M. M. C.; Nathwani, B.; Ryu, J. H.; Chou, L. Y. T.; Vinther, M.; Li, W. A.; Anastassacos, F. M.; Mooney, D. J.; Shih, W. M. Oligolysine-Based Coating Protects DNA Nanostructures from Low-Salt Denaturation and Nuclease Degradation. Nat. Commun. 2017, 8 (May), 1-9.

(22) Van de Cauter, L.; Fanalista, F.; van Buren, L.; De Franceschi, N.; Godino, E.; Bouw, S.; Danelon, C.; Dekker, C.; Koenderink, G. H.; Ganzinger, K. A. Optimized CDICE for Efficient Reconstitution of Biological Systems in Giant Unilamellar Vesicles. bioRxiv 2021, DOI: $10.1101 / 2021.02 .24 .432456$.

(23) Hanselmann, R.; Burchard, W.; Lemmes, R.; Schwengers, D. Characterization of DEAE-Dextran by Means of Light Scattering and Combined Size-Exclusion Chromatography/Low-Angle Laser Light Scattering/Viscometry. Macromol. Chem. Phys. 1995, 196 (7), 22592275.

(24) Görlich, D.; Henklein, P.; Laskey, R. A.; Hartmann, E. A 41 Amino Acid Motif in Importin- $\alpha$ Confers Binding to Importin- $\beta$ and Hence Transit into the Nucleus. EMBO J. 1996, 15 (8), 1810-1817.

(25) Baykal-Caglar, E.; Hassan-Zadeh, E.; Saremi, B.; Huang, J. Preparation of Giant Unilamellar Vesicles from Damp Lipid Film for Better Lipid Compositional Uniformity. Biochim. Biophys. Acta, Biomembr. 2012, 1818 (11), 2598-2604.

(26) Fick, A. Über Diffusion [Translated: (1995) "On Liquid Diffusion”. Journal of Membrane Science]. Ann. Phys. 1855, 170 (1), 59-86.

(27) Dechadilok, P.; Deen, W. M. Hindrance Factors for Diffusion and Convection in Pores. Ind. Eng. Chem. Res. 2006, 45 (21), 69536959. 
(28) Wente, S. R.; Rout, M. P. The Nuclear Pore Complex and Nuclear Transport. Cold Spring Harbor Perspect. Biol. 2010, 2 (10), a000562.

(29) Birnie, A.; Dekker, C. Genome-in-a-Box: Building a Chromosome from the Bottom Up. ACS Nano 2021, 15 (1), 111124.

(30) Douglas, S. M.; Marblestone, A. H.; Teerapittayanon, S.; Vazquez, A.; Church, G. M.; Shih, W. M. Rapid Prototyping of 3D DNA-Origami Shapes with CaDNAno. Nucleic Acids Res. 2009, 37 (15), 5001-5006.

(31) Kick, B.; Praetorius, F.; Dietz, H.; Weuster-Botz, D. Efficient Production of Single-Stranded Phage DNA as Scaffolds for DNA Origami. Nano Lett. 2015, 15 (7), 4672-4676.

(32) Wagner, T.; Merino, F.; Stabrin, M.; Moriya, T.; Antoni, C.; Apelbaum, A.; Hagel, P.; Sitsel, O.; Raisch, T.; Prumbaum, D.; Quentin, D.; Roderer, D.; Tacke, S.; Siebolds, B.; Schubert, E.; Shaikh, T. R.; Lill, P.; Gatsogiannis, C.; Raunser, S. SPHIRECrYOLO Is a Fast and Accurate Fully Automated Particle Picker for Cryo-EM. Commun. Biol. 2019, 2 (1), 1-13.

(33) Zivanov, J.; Nakane, T.; Forsberg, B.; Kimanius, D.; Hagen, W. J. H.; Lindahl, E.; Scheres, S. H. W. New Tools for Automated HighResolution Cryo-EM Structure Determination in RELION-3. eLife 2018, 7, e42166.

(34) Kremer, J. R.; Mastronarde, D. N.; McIntosh, J. R. Computer Visualization of Three-Dimensional Image Data Using IMOD. J. Struct. Biol. 1996, 116 (1), 71-76.

(35) Eisele, N. B.; Frey, S.; Piehler, J.; Görlich, D.; Richter, R. P. Ultrathin Nucleoporin Phenylalanine-Glycine Repeat Films and Their Interaction with Nuclear Transport Receptors. EMBO Rep. 2010, 11 (5), 366-372.

(36) Potma, E. O.; De Boeij, W. P.; Bosgraaf, L.; Roelofs, J.; Van Haastert, P. J. M.; Wiersma, D. A. Reduced Protein Diffusion Rate by Cytoskeleton in Vegetative and Polarized Dictyostelium Cells. Biophys. J. 2001, 81 (4), 2010-2019.

(37) Marquardt, D.; Heberle, F. A.; Greathouse, D. V.; Koeppe, R. E.; Standaert, R. F.; Van Oosten, B. J.; Harroun, T. A.; Kinnun, J. J.; Williams, J. A.; Wassall, S. R.; Katsaras, J. Lipid Bilayer Thickness Determines Cholesterols Location in Model Membranes. Soft Matter 2016, 12 (47), 9417-9428. 